\title{
Une force de portance élastohydrodynamique en matière molle
}

Thomas Salez $^{(1)}$ (thomas.salez@u-bordeaux.fr), Baudouin Saintyves ${ }^{(2)}$, Zaicheng Zhang ${ }^{(1)}$, Vincent Bertin ${ }^{(1)}$ et Abdelhamid Maali ${ }^{(1)}$

(1) Laboratoire Ondes et Matière d'Aquitaine (UMR CNRS 5798 et Université de Bordeaux), 351 Cours de la Libération, 33405 Talence Cedex

(2) High Concept Labs, MANA Contemporary, Chicago, IL (USA)

La friction est un phénomène

omniprésent, aux conséquences

majeures. Une stratégie pour

réduire les frottements entre deux

objets est d'intercaler une couche de fluide lubrifiant.

Pour des objets élastiques,

le couplage entre leur déformation et la pression due à l'écoulement engendre des effets non triviaux, tels que l'apparition d'une force de portance.

Dans ce contexte, le problème d'une particule libre dans un fluide et proche d'une paroi molle a été traité théoriquement avec des prédictions étonnantes.

La portance a été ensuite observée expérimentalement de manière indirecte, à diverses échelles. Enfin, sa première mesure directe et quantitative à l'échelle nanométrique a été réalisée.

\section{Le contact mou et humide}

Les contacts lubrifiés entre solides déformables sont très répandus dans la nature et l'industrie. Leur riche histoire en science et en ingénierie implique des problématiques et des échelles de longueur et de temps très variées. Les exemples incluent : les glissements de terrain catastrophiques en géologie mettant en jeu des écoulements confinés dans des sols poroélastiques (c'est-à-dire poreux et élastiques comme une éponge), l'aquaplaning des pneus en caoutchouc sur routes humides, les roulements à billes industriels (métalliques, mais se déformant aux grandes vitesses mises en jeu), les articulations synoviales et cartilagineuses, le mouvement cellulaire dans les vaisseaux sanguins ou dans des dispositifs microfluidiques artificiels... Ces exemples impliquent invariablement les phénomènes de frottement et d'adhésion, mais aussi un couplage dit "élastohydrodynamique " (EHD) entre l'écoulement du lubrifiant et la déformation des parois. Récemment, un tel couplage EHD lubrifié a attiré l'attention dans le contexte de la matière molle confinée, et pourrait bien jouer un rôle crucial dans le mouvement de diverses entités physiologiques et biologiques. Par exemple, les articulations osseuses des mammiferes mettent probablement en jeu l'interaction fine entre les cartilages mous et le liquide synovial, ce qui pourrait expliquer leur coefficient de friction effectif remarquablement bas. En outre, grâce à l'existence du couplage EHD, l'appareil à forces de surface (SFA) et le microscope à force atomique (AFM) permettent de sonder l'élasticité des matériaux souples et fragiles, en évitant tout contact adhésif invasif qui pourrait altérer leurs propriétés [1].

\section{Force de portance émergente en matière molle confinée}

Traditionnellement, les forces de portance, qui maintiennent un avion en l'air, résultent d'écoulements inertiels (c'est-àdire liés à l'accélération du fluide) autour des ailes. Cependant, dans le cadre du couplage EHD lubrifié introduit ci-dessus, une nouvelle force de portance a été prédite théoriquement. Cette dernière émerge pour des écoulements visqueux et confinés, où l'inertie est négligeable, à condition que les parois soient déformables. Même si l'idée d'une portance EHD est probablement antérieure, au travers de l'étude de matériaux industriels plus rigides, elle a été remise à l'ordre du jour dans le contexte de la matière molle dans les années 1990 [2].

Essentiellement, tout objet symétrique se déplaçant dans un fluide visqueux et le long d'une paroi élastique est repoussé de celle-ci par une force normale engendrée dynamiquement. Cette dernière résulte d'une brisure de la symétrie du contact intime, et donc de l'écoulement confiné associé, en raison de la déformation de la paroi (fig. 1). L'écoulement sous l'objet engendre une surpression à l'avant qui comprime le substrat élastique, et une 

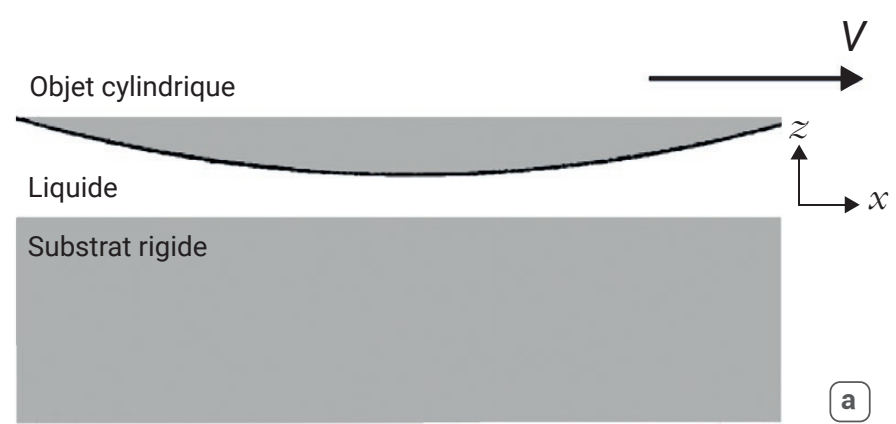

a
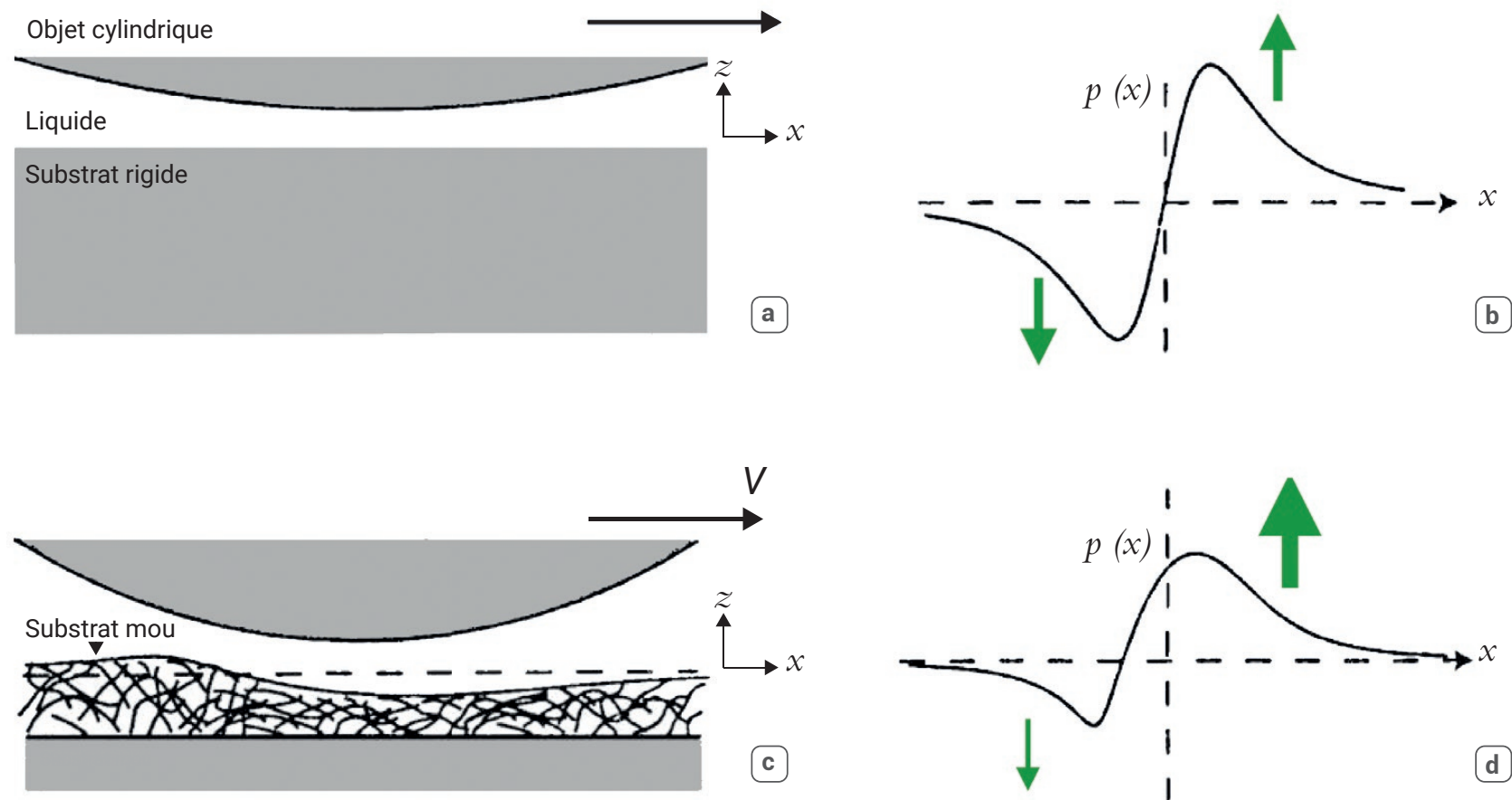

1. Principe de la force de portance élastohydrodynamique. Au voisinage d'un substrat rigide (a), le champ de pression $p(x)$ induit dans le liquide visqueux par le mouvement tangentiel d'un cylindre rigide se déplaçant à la vitesse $V$ est antisymétrique dans la direction tangentielle $x$ (b). En d'autres termes, la pression repousse autant le cylindre vers le haut à l'avant qu'elle ne le tire vers le bas à l'arrière (flèches vertes). Il en résulte une force normale (intégrale de la pression le long de $\mathrm{x}$ ) nulle dans la direction z. En revanche, un substrat mou (c) est déformé par le champ de pression. Ce dernier perd alors sa symétrie (d). Ainsi, les pressions à l'avant et à l'arrière du cylindre ne se compensent plus (flèches vertes). Par conséquent, l'intégrale selon $\mathrm{x}$ du champ de pression devient strictement positive, ce qui se traduit par une force normale pointant vers le haut : la portance élastohydrodynamique (image adaptée de [4]).

dépression à l'arrière qui, à l'inverse, attire le substrat vers l'objet. Cette asymétrie de la déformation induit une asymétrie de l'écoulement. Ainsi, comme pour la portance aérodynamique créée par l'asymétrie du flux d'air autour d'une aile d'avion, il se dégage ici aussi une portance. Cependant, son origine est élastohydrodynamique cette fois. De plus, à mesure que l'espace entre l'objet et la paroi souple est réduit, la force de portance augmente.

Ce mécanisme intrigant a été exploré théoriquement en détails, et généralisé au mouvement de vésicules molles le long d'une paroi rigide [3], ainsi que pour différents milieux élastiques et différentes géométries [4]. En outre, la brisure de symétrie sous-jacente du contact lubrifié n'est pas l'apanage de la seule élasticité. D'autres propriétés peuvent produire une portance analogue, telles que la capillarité de l'interface, une inhomogénéité de glissement, la géométrie du solide (même rigide)... Soulignons enfin que le mécanisme de portance décrit ici est distinct d'autres effets de portance mettant en jeu l'inertie, le caractère viscoélastique du fluide lui-même et/ou les variations spatiales de l'écoulement extérieur.

\section{Une zoologie de type inertiel contre-intuitive}

Dans les premières descriptions théoriques citées ci-dessus, la vitesse de la particule et l'épaisseur de la couche fluide sont supposées constantes et imposées de l'extérieur. Le cas d'une particule libre est cependant important en pratique. Dans ce contexte, nous avons prédit théoriquement une zoologie de trajectoires originales pour le cas d'un cylindre libre immergé dans un fluide visqueux, et qui peut simultanément sédimenter (tomber sous l'effet de la gravité), glisser ou rouler près d'un mur mou [5]. L'approche a consisté à résoudre les équations de l'hydrodynamique en confinement. Il a ainsi été possible de calculer d'abord les champs de vitesse et de pression dans le fluide dans le cas d'une paroi rigide, puis d'évaluer leurs modifications pour des parois élastiques faiblement déformables. Par intégration des contraintes résultantes à la surface du cylindre, les forces et couples émergents ont pu être calculés.

Le couplage entre déformation élastique et écoulement confiné ajoute des nonlinéarités qui modifient drastiquement la dynamique. Nous avons en effet observé des comportements très intrigants, comme : (i) un phénomène de "succion élastohydrodynamique ", où le cylindre sédimente de manière accélérée vers le mur ; 


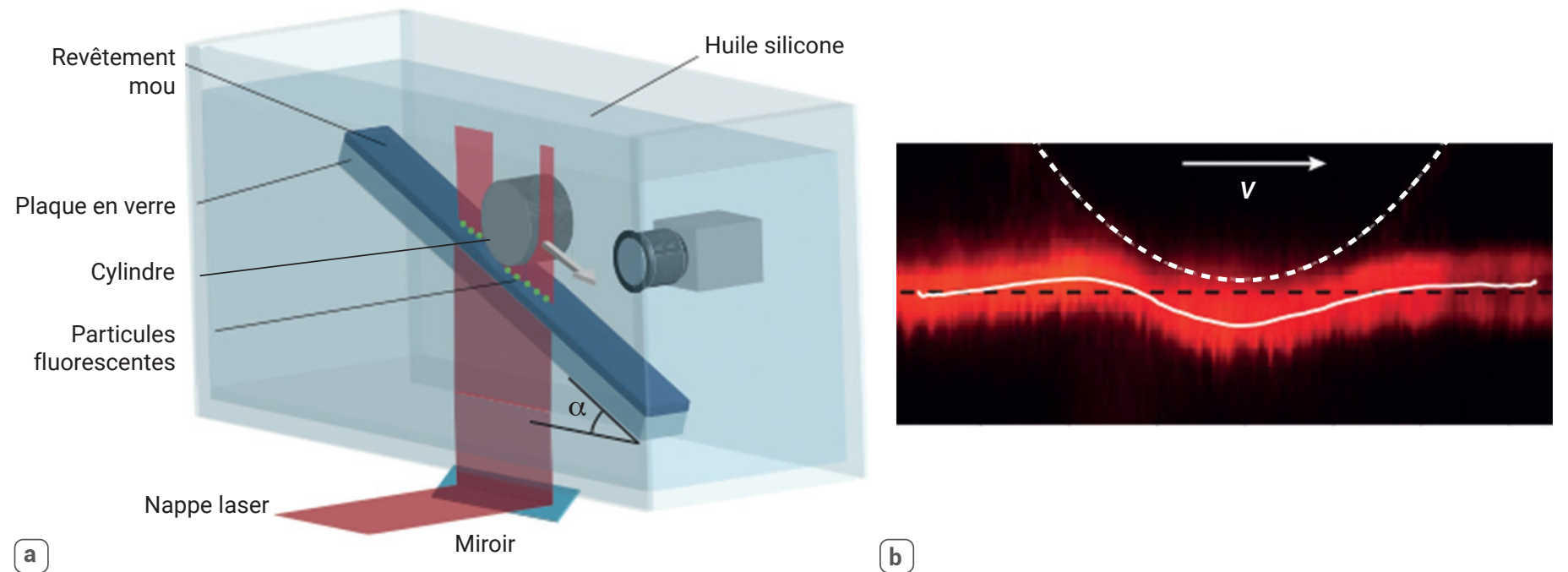

2. Montage expérimental macroscopique.

(a) Un cylindre rigide plongé dans un bain d'huile silicone glisse le long d'un plan incliné recouvert d'une fine couche élastique. Des particules fluorescentes encastrées à la surface de cette dernière permettent d'observer sa déformation à l'aide d'un laser et d'une caméra.

(b) Vue latérale redressée. La ligne pointillée blanche indique le profil du cylindre se déplaçant à la vitesse tangentielle V. La ligne en tirets noirs correspond à la surface non déformée de la couche élastique. Le trait plein blanc correspond à la moyenne du signal fluorescent (rouge), révélant le profil de la déformation. Les paramètres expérimentaux sont ici : épaisseur de la couche élastique $h=1,5 \mathrm{~mm}$, module élastique de cisaillement de cette couche $\mathrm{G}=65 \mathrm{kPa}$, viscosité du l'huile $\eta=1 \mathrm{~Pa}$.s, rayon du cylindre $\mathrm{R}=12,7 \mathrm{~mm}$, densité du cylindre $\rho=8510 \mathrm{~kg} \cdot \mathrm{m}^{-3}$ et angle $\alpha=11^{\circ}$.

$\gg>$

(ii) un effet de type Magnus (c'est-à-dire une force de portance hydrodynamique exercée sur un objet en rotation, comme une balle de tennis [6]), où la portance est contrôlée par la rotation propre du cylindre ;

(iii) un effet de type "boomerang " (ou "rétro " au billard), avec un retour en arrière spontané du cylindre ;

(iv) une inversion du sens de roulement ;

(v) ou encore un phénomène d'aquaplanage spontané et auto-entretenu...

Fait intéressant, ces effets sont souvent rencontrés dans des écoulements inertiels, sous amortis, généralement associés à des objets gros et rapides. A contrario, et comme pour la portance EHD décrite plus haut, ils sont ici engendrés à petite échelle, à basse vitesse et sans accélération du fluide, par la seule présence de frontières élastiques voisines. Ceci pourrait avoir des implications importantes en biologie, et on pourrait alors même envisager de " jouer au tennis » avec une cellule...

Cependant, malgré les idées et avancées théoriques discutées précédemment, des preuves expérimentales manquaient jusqu'à très récemment.

\section{Observation macroscopique indirecte : réduction de la friction effective}

Afin de sonder expérimentalement l'hypothétique force de portance EHD, nous avons étudié le mouvement libre et lubrifié d'un cylindre pesant le long d'une paroi molle [7]. Le dispositif expérimental est constitué d'un plan incliné rigide en verre, recouvert d'une fine couche élastique d'élastomère (polydiméthylsiloxane, PDMS) ou de gel (polyacrylamide, PAA), le tout immergé dans un récipient rempli d'huile silicone de viscosité contrôlée (fig. 2a).

À l'aide de ce dispositif, nous avons alors observé et étudié la chute de cylindres de tailles et matériaux divers, tout en faisant varier le module élastique et l'épaisseur du revêtement mou. En comparaison avec le cas d'une surface rigide (ici le verre sans revêtement), nous avons observé que la vitesse tangentielle $V$ du cylindre augmente significativement pour une surface molle (fig. 3a), ce qui correspond à une diminution du frottement effectif. La vitesse observée, et donc le frottement, apparaissent relativement constants dans l'espace et le temps, indiquant la suppression du phénomène de " coller-glisser » caractéristique du frottement sec entre deux solides.
De surcroit, lors du passage du cylindre le long de la paroi, le revêtement mou est déformé de manière asymétrique (fig. 2b), ce qui permet de confirmer l'existence d'un couplage EHD (fig. 1c-d).

Les résultats expérimentaux corroborent les prédictions théoriques exposées précédemment, dans le cas de faibles déformations de la surface. La force de portance s'oppose à la composante normale du poids, ce qui explique pourquoi le cylindre lévite lors de sa chute. La puissance motrice de la composante tangentielle du poids est quant à elle entièrement dissipée par l'écoulement visqueux confiné sous le cylindre, entrainant un phénomène de type aquaplanage à vitesse constante. La théorie prédit une vitesse tangentielle qui diminue avec le module élastique de cisaillement, ce qui a été vérifié quantitativement pour une large gamme de paramètres (fig. 3b) : épaisseur de la couche élastique $h=0,1$ à $2 \mathrm{~mm}$, module de cisaillement élastique de cette couche $G=8$ à $300 \mathrm{kPa}$, viscosité de l'huile $\eta=0,01$ à 100 Pa.s, angle d'inclinaison $\alpha=10$ à $40^{\circ}$, densité du cylindre $\rho=2240$ à $8510 \mathrm{~kg} \cdot \mathrm{m}^{-3}$ et rayon du cylindre $R=6,35$ à $12,7 \mathrm{~mm}$. 
Cependant, lorsque les gels deviennent très mous (c'est-à-dire lorsque le module élastique de cisaillement devient inférieur à $10 \mathrm{kPa}$ ), donc à grande déformation, la théorie asymptotique précédente n'est plus vérifiée, et la vitesse atteint un maximum, puis diminue même à nouveau dans certains cas (fig. 3a). Ceci suggère l'existence d'un mécanisme de saturation, voire d'une rigidité optimale pour réduire le frottement effectif. La saturation pourrait par exemple être due à un nivellement de la déformation élastique, qui ne pourrait excéder l'épaisseur du film de fluide.

Notons enfin que le même dispositif expérimental nous a permis d'étudier en détails la rotation et le couple EHD émergeant spontanément sur le cylindre lors de sa translation. Nous ne discuterons pas cet aspect ici, car l'idée est analogue au cas de la force de portance.

\section{Observations indirectes complémentaires : systèmes biomimétiques et élancés}

Par la suite, deux études dans la communauté naissante autour de la problématique du contact EHD en matière molle ont confirmé l'existence de la force de portance, et ce de manière indirecte également.

Tout d'abord, une équipe grenobloise a réalisé le suivi optique du mouvement d'une microparticule transportée par un écoulement, dans un canal microfluidique dont les parois avaient été recouvertes au préalable d'une brosse de polymère biomimétique [8]. La trajectoire de la particule s'écarte systématiquement des parois, avec une loi en accord avec le modèle de portance EHD. Cette étude expérimentale révèle ainsi pour la première fois l'impor- tance de cette force de portance dans des contextes microfluidiques pertinents pour la biologie.

Une seconde étude a consisté à observer la sédimentation gravitationnelle d'une bille millimétrique le long d'une membrane verticale tendue. Ses auteurs ont observé une importante dérive perpendiculairement à la membrane et en s'en écartant, révélant ainsi la grande amplification de la force de portance pour les frontières très déformables associées aux géométries élancées [9]. De nouveau, il existe un intérêt potentiel pour la biologie, puisque les membranes y sont omniprésentes.

Néanmoins, si cette littérature expérimentale récente corrobore l'existence de la force de portance EHD, ainsi que son importance potentielle à petite échelle et en biophysique, une mesure de force, directe et à l'échelle nanométrique, faisait alors encore défaut.

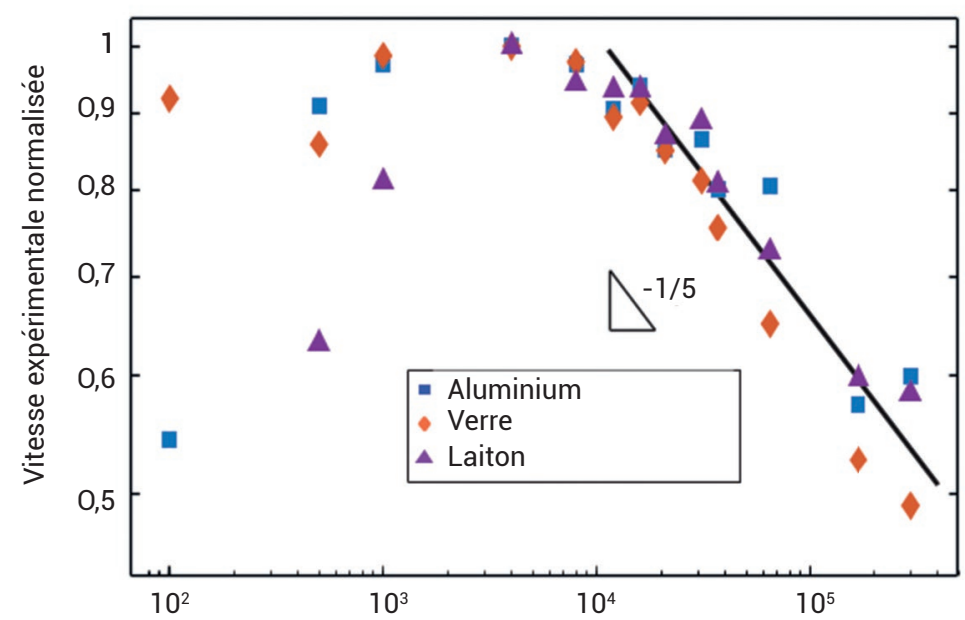

a

Module de cisaillement élastique $(\mathrm{Pa})$

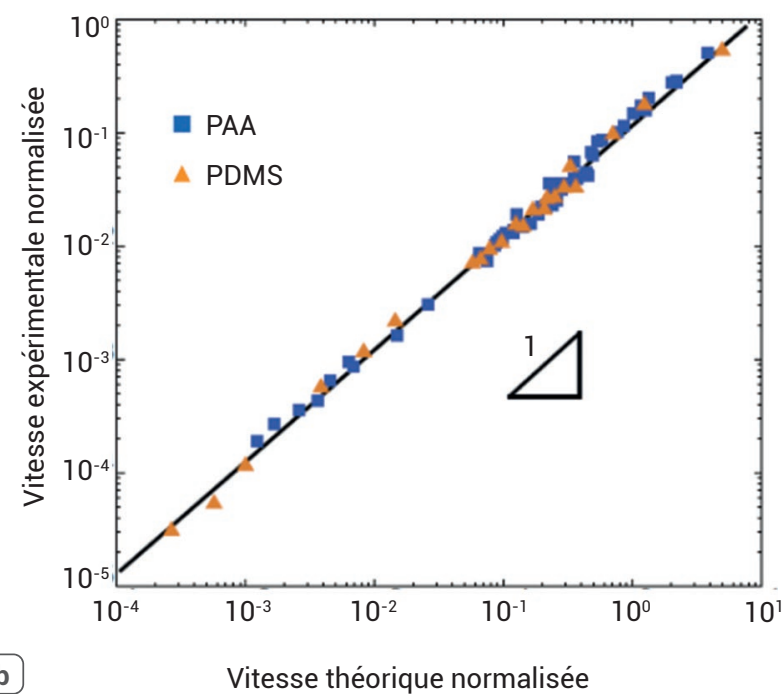

3. Résultat macroscopique : réduction de la friction effective.

(a) Vitesse tangentielle expérimentale du cylindre en fonction du module de cisaillement de la couche élastique, pour trois cylindres de matériaux différents. L'axe vertical est normalisé par la vitesse maximale mesurée pour chaque cylindre. Les paramètres expérimentaux communs sont ici : épaisseur de la couche élastique $h=600 \mu \mathrm{m}$, viscosité de l'huile $\eta=1 \mathrm{~Pa}$.s et angle $\alpha=11^{\circ}$. Les paramètres spécifiques sont les suivants : cylindre 1 (aluminium) : densité $\rho=2720 \mathrm{~kg} \cdot \mathrm{m}^{-3}$, rayon $R=12,7 \mathrm{~mm}$; cylindre 2 (verre) : $\rho=2240 \mathrm{~kg} \cdot \mathrm{m}^{-3}, \mathrm{R}=9,5 \mathrm{~mm}$; cylindre 3 (laiton) : $\rho=8510 \mathrm{~kg} \cdot \mathrm{m}^{-3}, \mathrm{R}=6,35 \mathrm{~mm}$. La ligne en trait plein a une pente de $-1 / 5$ dans cette représentation log-log. En comparaison, le cas d'un substrat rigide donnerait une vitesse quasi nulle (résultat non présenté ici).

(b) Vitesse tangentielle expérimentale du cylindre en fonction de la vitesse théorique, pour l'ensemble des expériences réalisées avec des couches d'élastomères (polydiméthylsiloxane, PDMS) et d'hydrogels (polyacrylamide, PAA). Les deux axes sont normalisés par une même vitesse de chute libre de référence. La ligne en trait plein a une pente de 1 dans cette représentation log-log. 


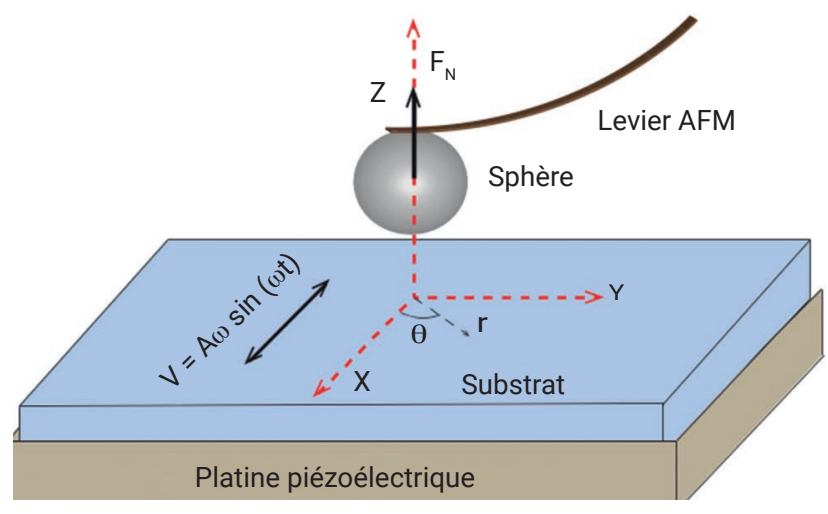

a

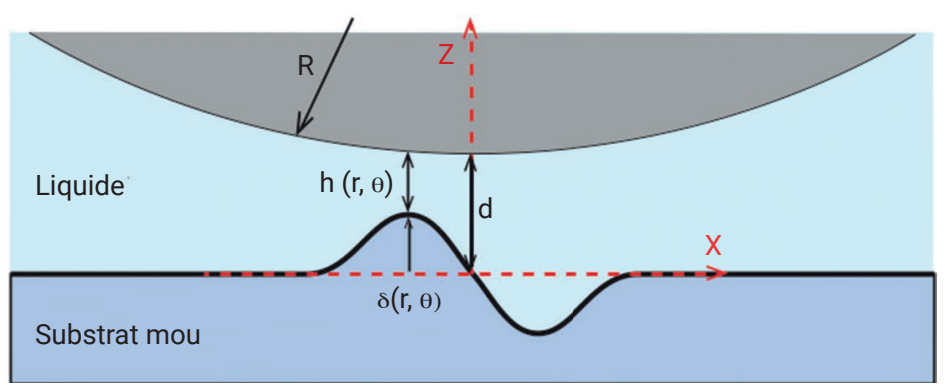

(b)

4. Montage expérimental microscopique. Vues d'ensemble (a) et rapprochée (b). Un substrat mou est fixé sur une platine piézoélectrique de translation oscillant transversalement au cours du temps $t$, dans la direction $x$, avec la pulsation $\omega$, l'amplitude spatiale $A$ et la vitesse $V$. Une sphère rigide de silice de rayon micrométrique $\mathrm{R}$ est collée sur un levier de microscope à force atomique (AFM) et immergée dans un liquide visqueux à proximité du substrat. La force normale $F_{N}$ exercée sur la sphère dans la direction $z$, à une distance nanométrique d de la surface, est directement mesurée à partir de la déflexion du levier (de raideur connue). Le champ de déformation normal $\delta$ induit à la surface libre du substrat par l'écoulement visqueux modifie le profil d'épaisseur h du liquide confiné, et donc les contraintes hydrodynamiques qui s'exercent sur la sphère (voir la figure 1).

\section{Mesure microscopique directe de la portance élastohydrodynamique}

Pour effectuer une mesure quantitative et directe de la force de portance à petite échelle, nous avons collé une sphère, de rayon $R=60 \mu \mathrm{m}$, à la pointe d'un levier de microscope à force atomique (AFM) de raideur $k=210 \mathrm{mN} \cdot \mathrm{m}^{-1}$. Nous l'avons ensuite placée dans un liquide visqueux (huile silicone ou alcool), près d'un substrat élastomérique (polydiméthylsiloxane, PDMS) déformable mais incompressible, d'épaisseur de l'ordre de $30 \mu \mathrm{m}$ et oscillant latéralement (fig. 4a) [10]. Ce faisant, un couplage EHD est induit (fig. 4b). Des mesures de la force normale $F_{\mathrm{N}}$ moyennée dans le temps (c'est-à-dire $F=<F_{\mathrm{N}}>$ ) ont été obtenues pour plusieurs distances sphère-substrat $d$ entre $1 \mathrm{~nm}$ et $10 \mu \mathrm{m}$, et dans diverses conditions expérimentales : fréquence d'oscillation $f=\omega /(2 \pi)=25$ à $50 \mathrm{~Hz}$, amplitude d'oscillation $A=3,6$ à $36 \mu \mathrm{m}$, module de cisaillement élastique du substrat $G=98$ à $485 \mathrm{kPa}$ et viscosité du liquide $\eta=14$ à $96 \mathrm{mPa} . \mathrm{s}$.

Alors qu'aux abords d'une surface rigide (silicium sans revêtement élastique), la force mesurée est nulle, une force de portance de l'ordre de la dizaine de nanonewtons est mesurée pour des substrats mous (fig. 5a). La force suit une loi de puissance avec la distance, d'exposant $-5 / 2$ (voir l'encart sur la figure 5a), ce qui est une caractéristique typique du couplage EHD dans le cas des substrats épais et incompressibles, pour lesquels on attend la loi d'échelle : $F_{\mathrm{N}} \sim\left(\eta^{2} V^{2} / G\right) .(R / d)^{5 / 2}$ [4]. De surcroit, à une distance $d$ donnée, plus la paroi est molle (c'est-à-dire plus $G$ est faible), plus la force est grande, comme attendu. De même, les expériences montrent bien que la force augmente avec la vitesse $V$ du substrat (et donc du fluide).

Toutes ces mesures peuvent être regroupées sur une seule courbe maitresse (fig. 5b), donnée par la loi d'échelle précédente. En outre, nous avons pu calculer exactement le préfacteur de cette loi d'échelle, ce qui permet de capturer sans paramètre ajustable l'ensemble des données, à faible complaisance élastique (c'est-à-dire grand module élastique) ou large distance. Enfin, à courte distance, les observations indiquent une saturation de l'effet de portance (voir l'encart de la figure 5a), ce qui fait écho à la saturation précédemment observée indirectement via la mesure macroscopique de la friction effective (fig. 3a).

Nous soulignons qu'une mesure indépendante de la force de portance EHD a été réalisée de manière concomitante par une autre équipe bordelaise, pour un système différent : des couches de microgels compressibles étudiées à l'aide d'un appareil à forces de surface (SFA) [11].

\section{Perspectives}

L'ensemble de ces questions et résultats marque l'émergence d'une nouvelle communauté en France et dans le monde, autour de la problématique du contact élastohydrodynamique en matière molle. Cette activité engendre de nouvelles idées pour aborder des phénomènes aussi divers que le vieillissement des articulations cartilagineuses ou l'origine des glissements de terrain. Les avancées récentes laissent entrevoir des perspectives d'applications fascinantes, vers les lubrifiants intelligents et l'ingénierie fonctionnalisée de la friction (par exemple, pour les pistons des moteurs), ou encore vers la rhéologie locale non invasive des nano/biomatériaux et des revêtements fragiles de surfaces.

Dans un futur proche, l'exploration expérimentale de la zoologie de comportements de type inertiel décrite ci-dessus semble être une tâche pertinente, ainsi que l'incorporation de caractéristiques complexes (par exemple viscoélastiques et poroélastiques) à celle-ci. Les cartilages, avec leurs propriétés de friction incroyables et encore mal comprises, pourraient être revisités sous l'angle élastohydrodynamique, de façon à mieux les comprendre et pouvoir concevoir de nouvelles prothèses biomimétiques, par exemple. Outre l'élasticité, d'autres mécanismes de brisure de symétrie du contact lubrifié pourraient être explorés expérimentalement, tels que : 

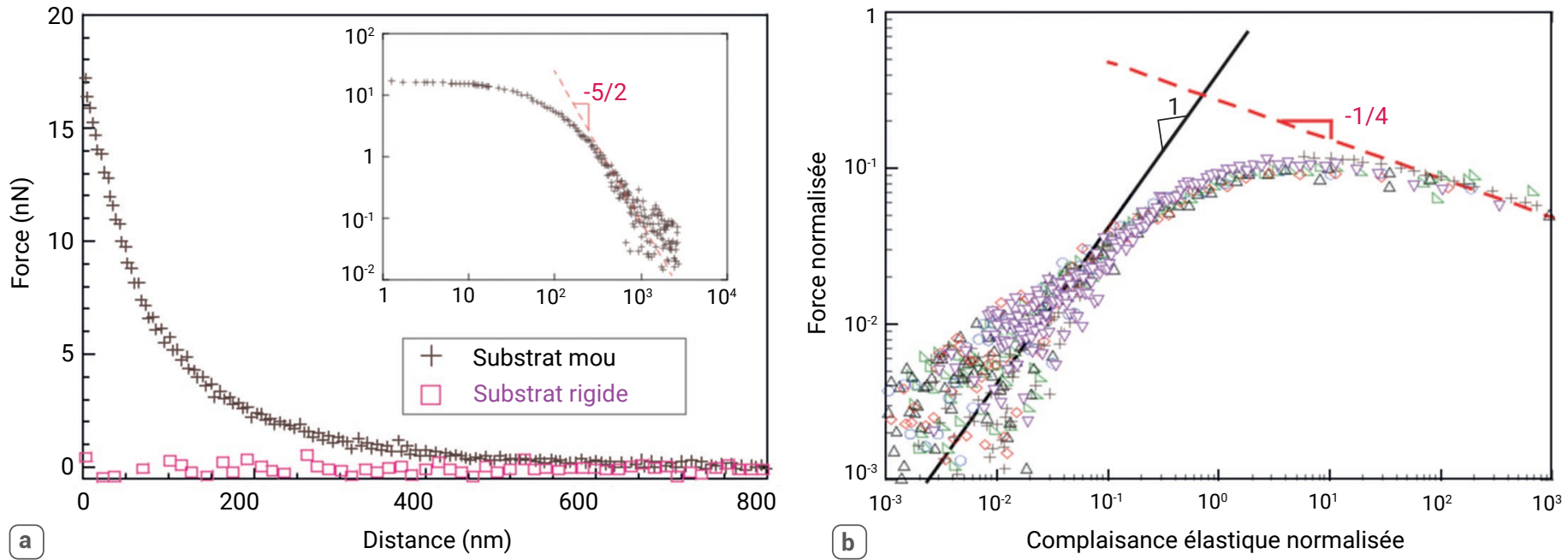

5. Résultat microscopique : mesure directe de la force de portance élastohydrodynamique. (a) Moyenne temporelle $F$ de la force normale $F_{N} s^{\prime} e x e r c ̧ a n t$ sur la sphère (voir la figure 4) en fonction de la distance $d$ au substrat, pour des substrats rigide (silicium) et mou (PDMS), et pour un jeu de paramètres (fréquence, amplitude, viscosité et rayon de la sphère) fixé. L'encart montre une représentation log-log des données pour le cas mou où la ligne en tirets est de pente $-5 / 2$, caractéristique de l'exposant de la loi de puissance attendue dans le cas d'un substrat élastique incompressible semi infini [3]. (b) Force normale moyenne normalisée par une force de référence en fonction de la complaisance élastique (c'est-à-dire l'inverse du module élastique) normalisée par une complaisance de référence, en échelles logarithmiques, résultant des données force-distance (voir (a)), pour toutes les expériences réalisées et paramètres explorés dans cette étude. La ligne noire en trait plein de pente 1 correspond à la prédiction théorique asymptotique à faible complaisance (ou grande distance), sans paramètre ajustable. La ligne rouge en tirets indique une loi de puissance empirique d'exposant - $1 / 4$ dans le régime de forte complaisance (ou faible distance).

i) des inhomogénéités de friction ; ou ii) des réponses capillaires propres aux liquides et aux gels très mous, pour n'en nommer que quelques-uns.

En outre, moduler spatialement et de façon optimisée la complaisance élastique pourrait être un moyen, au travers des couplages EHD résultants, d'ajuster le frottement de manière efficace (par exemple pour les sports de glisse) et de diriger le transport microfluidique de particules, dans l'esprit de la durotaxie (la tendance à se déplacer dans le sens d'un gradient de rigidité) des cellules biologiques.

Enfin, des phénomènes collectifs de type " effet Cheerios » (c'est-à-dire l'attraction capillaire entre des objets flottant à la surface libre d'un liquide), engendrés par l'apparition d'interactions effectives, pourraient être abordés dans un contexte de couplage EHD. Ceci permettrait, par exemple, de développer une microingénierie fine pour la rhéologie des suspensions molles.

Les résultats présentés dans cet article sont le fruit d'une collaboration internationale incluant :

L. Mahadevan, T. Jules, B. Rallabandi, C. Schönecker, H. Stone, M. Arshad, É. Raphaël et J. Ault.

Nous les remercions vivement.

\section{Références}

1• R. Villey et al., « Indenter du verre avec un liquide ? ", Reflets de la Physique, 43 (2015) 38.

2•K. Sekimoto et L. Leibler, "A mechanism for shear thickening of polymer-bearing surfaces: elasto-hydrodynamic coupling", Europhysics Letters, 23 (1993) 113.

$3 \cdot \mathrm{J}$. Beaucourt et al., "Optimal lift force on vesicles near a compressible substrate", Europhysics Letters, 67 (2004) 676.

4. J. M. Skotheim et L. Mahadevan, "Soft lubrication: the elastohydrodynamics of nonconforming and conforming contacts", Physics of Fluids, 17 (2005) 092101.

5. T. Salez et L. Mahadevan, "Elastohydrodynamics of a sliding, spinning and sedimenting cylinder near a soft wall", Journal of Fluid Mechanics, 779 (2015) 181.

6• G. Dupeux et al., " Le football et ses trajectoires », Reflets de la Physique, 28 (2012) 10.

7• B. Saintyves et al., "Self-sustained lift and low friction via soft lubrication",

Proceedings of the National Academy of Sciences of the USA, 113 (2016) 5847.

8. H. S. Davies et al., "Elastohydrodynamic lift at a soft wall", Phys. Rev. Lett., 120 (2018) 198001.

9. B. Rallabandi et al., "Membrane-induced hydroelastic migration of a particle surfing its own wave", Nature Physics, 14 (2018) 1211.

10 Z. Zhang et al., "Direct measurement of the elastohydrodynamic lift force at the nanoscale", Phys. Rev. Lett., 124 (2020) 0504502.

11 • P. Vialar et al., "Compliant surfaces under shear: elastohydrodynamic lift force", Langmuir, 35 (2019) 15605. 